\title{
BERKELEY UXO DISCRIMINATOR (BUD)
}

\author{
Erika Gasperikova, Lawrence Berkeley National Laboratory, Berkeley, CA \\ $J$. Torquil Smith, Lawrence Berkeley National Laboratory, Berkeley, CA \\ H. Frank Morrison, Lawrence Berkeley National Laboratory, Berkeley, CA \\ Alex Becker, Lawrence Berkeley National Laboratory, Berkeley, CA
}

\begin{abstract}
The Berkeley UXO Discriminator (BUD) is an optimally designed active electromagnetic system that not only detects but also characterizes UXO. The system incorporates three orthogonal transmitters and eight pairs of differenced receivers. It has two modes of operation: (1) search mode, in which BUD moves along a profile and exclusively detects targets in its vicinity, providing target depth and horizontal location, and (2) discrimination mode, in which BUD, stationary above a target, from a single position, determines three discriminating polarizability responses together with the object location and orientation. The performance of the system is governed by a target size-depth curve. Maximum detection depth is $1.5 \mathrm{~m}$. While UXO objects have a single major polarizability coincident with the long axis of the object and two equal transverse polarizabilities, scrap metal has three different principal polarizabilities. Our results clearly show that there are very clear distinctions between symmetric intact UXO and irregular scrap metal, and that BUD can resolve the intrinsic polarizabilities of the target. The field survey at the Yuma Proving Ground in Arizona showed excellent results within the predicted sizedepth range.
\end{abstract}

\section{Introduction}

The next generation of truly portable Active Electromagnetic Systems (AEM) for UXO detection and characterization must be able to quickly determine the location, size, and symmetry properties of a suspected UXO. The Berkeley UXO discriminator (BUD) (Figure 1a) is such a system. It is a multiple transmitter-multiple receiver system designed to detect UXO in the $20 \mathrm{~mm}$ to $155 \mathrm{~mm}$ size range (Figure 1b) for depths between 0 and $1.5 \mathrm{~m}$, and to characterize them in a depth range from 0 to $1.1 \mathrm{~m}$.

The BUD comprises of three orthogonal transmitters that "illuminate" a target with fields in three independent directions in order to stimulate the three polarization modes that, in general, characterize the target EM response. In addition, the BUD uses eight pairs of differenced receivers for response recording. Receiver coils are placed horizontally along the two diagonals of the upper and lower planes of the two horizontal transmitter loops. The receiver coil pairs are located on symmetry lines through the center of the system, with each pair seeing identical fields during the on-time of the pulse in all of the transmitter coils. They are wired in opposition to produce zero output during the ontime of the pulses in three orthogonal transmitters. This configuration dramatically reduces noise in the measurements by canceling the background electromagnetic fields (these fields are uniform over the scale of the receiver array and are consequently nulled by the differencing operation), and by canceling the noise contributed by the tilt of the receivers in the Earth's magnetic field, and greatly enhances receivers sensitivity to the gradients of the target response. 
The BUD has two modes of operation: (1) search mode, in which it moves along a profile and exclusively detects targets in its vicinity, providing target depth and horizontal location, and (2) discrimination mode, in which BUD, stationary above a target, from a single position, determines three discriminating polarizability responses, together with the object location and orientation.

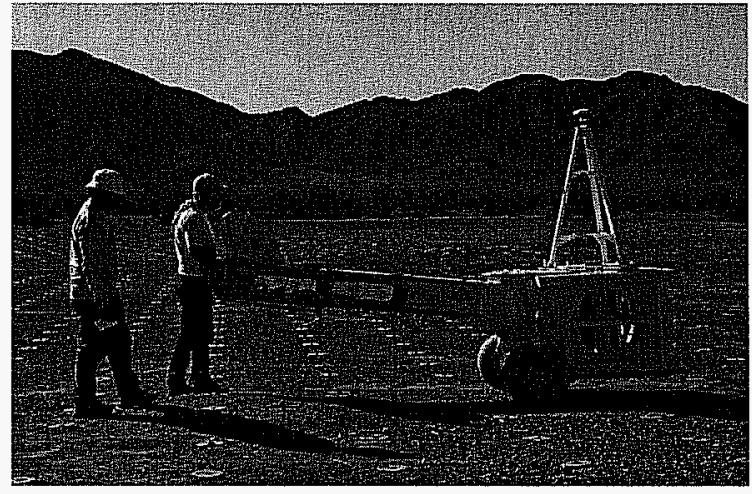

(a)

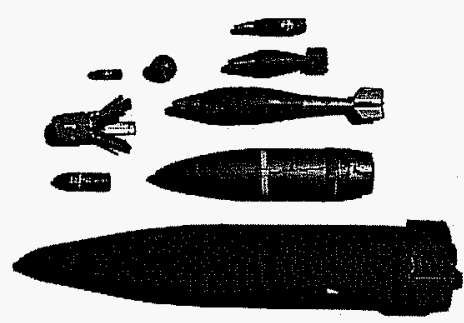

(b)

Figure 1: (a) Berkeley UXO Discriminator (BUD), (b) UXO size range.

A major advance in discriminating UXO from irregular scrap metal is a determination of the principal dipole polarizabilities of a target. The induced moment of a target depends on the strength of the transmitted inducing field. The moment normalized by the inducing field is the polarizability. Any UXO is expected to retain its fundamental shape (size, aspect ratio, and symmetry about its long axis) with perhaps minor distortion caused by impact. A near-intact UXO displays a single major polarizability coincident with the long axis of the object and two equal transverse polarizabilities. In contrast, metal scrap will have distinct polarizability signatures that cannot mimic those of elongated symmetric bodies. Roughly flat sheets will have dipolar responses approaching those of a highly flattened oblate spheroid (close to a loop response), twisted sheets a principal polarizability orthogonal to some equivalent plane through the sheet with small and highly irregular, minor axis polarizabilities, etc. These distinguishing polarizabilities, coupled with the size estimates and spatial sampling of the multiple receiver array, are more than enough to distinguish small scrap from deeper targets of concern. Figure 2 illustrates the discrimination capability of BUD both for UXO objects and scrap metal. Estimated principal polarizabilities are plotted as a function of time, with object images shown at the top right corner.

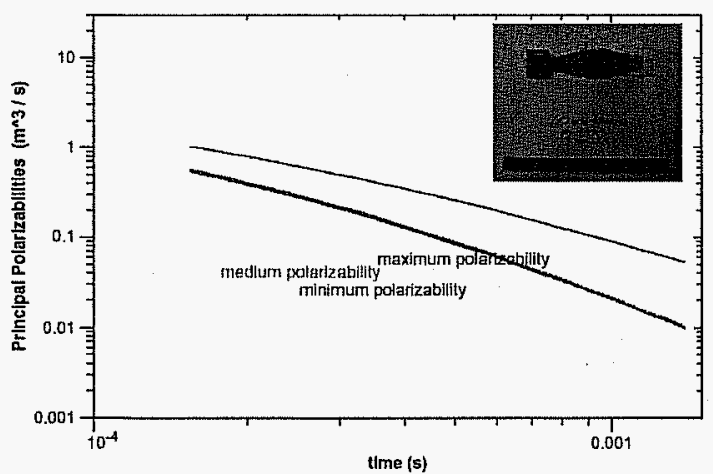

(a)

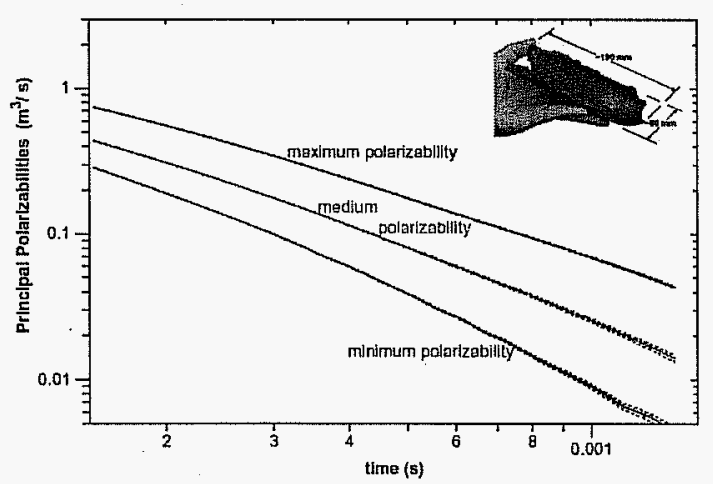

(b)

Figure 2. Inversion results for the principal polarizabilities of (a) UXO and (b) non-UXO (scrap metal). 
Whereas UXO objects have a single major polarizability coincident with the long axis of the object and two equal transverse polarizabilities (Figure 2a), scrap metal exhibits three distinct principal polarizabilities (Figure 2b). The object locations and orientations are recovered within a few percent of true values (not shown). These results clearly show that a multiple transmitter-multiple receiver system can resolve the intrinsic polarizabilities of a target, and that very clear distinctions exist between symmetric intact UXO and irregular scrap metal - distinctions that could not be made from the single fixed position of any prior UXO AEM system.

One important component of our new AEM system is an inversion algorithm that can determine target properties from measurements using the given transmitter-receiver configuration. At any given time, the response is inverted to yield the location $(x, y, z)$ of the target, its attitude, and its principal polarizabilities, which yield an apparent aspect ratio. Signal-to-noise estimates (or measurements) are interpreted in this inversion to yield error estimates on location, attitude, and polarizabilities. This inversion at a succession of times provides the polarizabilities as a function of time, which can in turn yield target size, true aspect ratio, and estimates of conductivity and permeability. The accuracy of these property estimates depends on the time window over which the polarizability measurements, and their accuracies, are known.

As described earlier, the response of a spheroid to uniform incident fields, as a function of time, is characterized by two equal polarizabilities from fields oriented in directions orthogonal (transverse) to the spheroid's axis of symmetry, and a third polarizability from fields aligned with its axis of symmetry. The response of an object with no symmetry (i.e., irregular scrap metal) has three independent principal polarizabilities. To interpret measured principal polarizabilities in terms of equivalent conducting magnetically permeable spheroids, we average the two principal polarizabilities that differ least from each other (over the duration of the measured response) and treat it as a transverse polarizability, and the third as an axial polarizability. These are inverted for major and minor spheroid diameters, conductivity $(\sigma)$, and relative permeability $\left(\mu_{\mathrm{r}}\right)$, using a general non linear optimization method (Smith, Allan, and Schultz, 1994; Smith and Morrison, 2005) to minimize the function of the squared, uncertainty normalized, residuals, with large residuals down weighted to reduce sensitivity to non-Gaussian errors. Within the inversion, the polarizability response of spheroids is approximated using the scaled-sphere approximation of Smith and Morrison (2006). The product $\sigma \cdot \mu_{\mathrm{r}}$ is, in general, poorly resolved, and thus

it is constrained to within a range encompassing copper and steel values. Uncertainties in major and minor diameters, the ratio $\sigma / \mu_{\mathrm{r}}$ and product $\sigma \cdot \mu_{\mathrm{r}}$, are estimated by propagating estimated errors in the input polarizabilities into these quantities, based on the sensitivity (Jacobian) of calculated data to changes in them.

\section{BUD Performance}

The dimensions of the transmitter control the field strength at depth, while spacing of receivers controls the accuracy of depth estimates. The detection performance of BUD is governed by a sizedepth curve shown in Figure 3a. This curve was calculated assuming that the receiver plane is $0.2 \mathrm{~m}$ above the ground. Figure $3 \mathrm{a}$ shows that, for example, BUD can detect an object with $0.1 \mathrm{~m}$ diameter down to a depth of $0.9 \mathrm{~m}$, with depth uncertainty of $10 \%$. Any objects buried at depth greater than 1.3 $\mathrm{m}$ will have a low probability of detection. 
The discrimination performance of BUD is governed by a size-depth curve shown in Figure $3 b$, once again assuming that the receiver plane is $0.2 \mathrm{~m}$ above the ground. Figure $3 \mathrm{~b}$ shows that, for example, BUD can discriminate an object with $0.1 \mathrm{~m}$ diameter down to a depth of $0.63 \mathrm{~m}$, with depth uncertainty of $10 \%$. Any objects buried at a depth greater than $0.9 \mathrm{~m}$ will have a low probability of discrimination. When the size-depth requirement (Figure 3) is satisfied, the polarizabilities are independent of the depth and orientation of the object. For large objects close to the system (above the red line in Figure $3 b$ ), the principal polarizability curves vary depending on the orientation of the object.

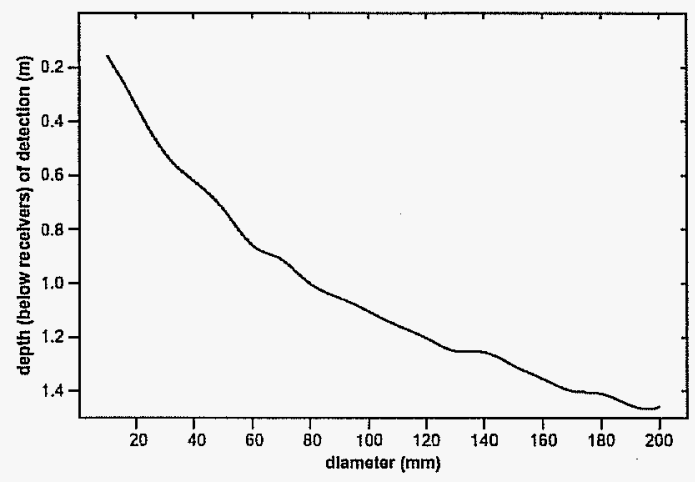

(a)

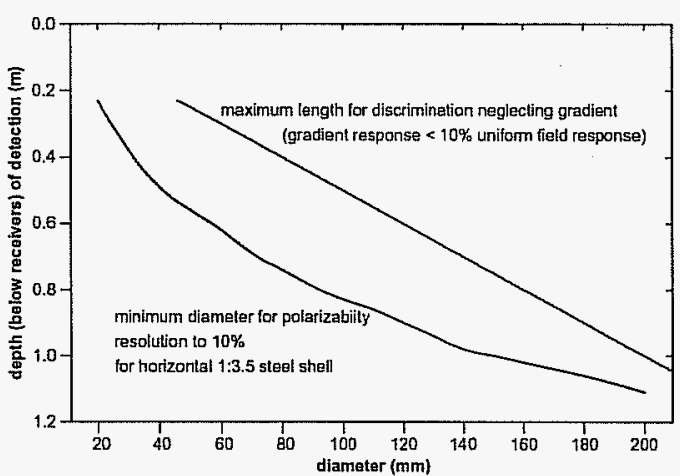

(b)

Figure 3. (a) BUD detection performance, assuming $10 \%$ uncertainty in location as a function of object diameter and depth with receivers $0.2 \mathrm{~m}$ above the ground, (b) BUD discrimination performance, assuming $10 \%$ uncertainty in location as a function of object diameter and depth with receivers $0.2 \mathrm{~m}$ above the ground.

\section{Field Survey Results}

We tested BUD by acquiring multicomponent data over the Calibration Grid and the Blind Test Grid at the Yuma Proving Ground. The Calibration Grid is a $30 \mathrm{~m}$ by $40 \mathrm{~m}$ area consisting of seventeen lanes and containing 132 UXO objects; the Blind Test Grid is a $1,600 \mathrm{~m}^{2}$ area containing 400 cells, each of which can be occupied by UXO, clutter, or both, or it can be empty. The Calibration Grid was surveyed using $1 \mathrm{~m}$ spacing along Lanes A through $\mathrm{K}$, and $0.5 \mathrm{~m}$ spacing along Lanes $\mathrm{L}$ and $\mathrm{M}$. The Blind Test Grid was surveyed using $0.5 \mathrm{~m}$ spacing along the lanes, and $1 \mathrm{~m}$ spacing between lanes.

A set of responses was collected over standardized targets in the Calibration Grid and a test pit. Data were differenced with a reference site measurement and then inverted for equivalent dipole polarizability and location using the method of Smith and Morrison (2004), but using the empirical distribution evolutionary algorithm of Smith, et al. (1994) (also, Smith and Morrison, 2005) in place of the downhill simplex method used by Smith and Morrison (2004). When the size-depth requirement (Figure 3 ) is satisfied, the polarizabilities are independent of the depth and orientation of the object. For large objects close to the system, the principal polarizabilities curves vary depending on the orientation of the object. Figure 4 shows estimated principal polarizabilities of four targets plotted as a function of time, with object images shown at the top right corner. While UXO objects have the single major polarizability coincident with the long axis of the object and two equal transverse polarizabilities (Figure $4 \mathrm{c}$ and $4 \mathrm{~d}$ ), the loop of wire has only a single major polarizability (minor axis polarizabilities are very small and highly irregular) (Figure 4a), and the 12-lb. shot put is characterized by three identical polarizabilties (Figure 4b). 

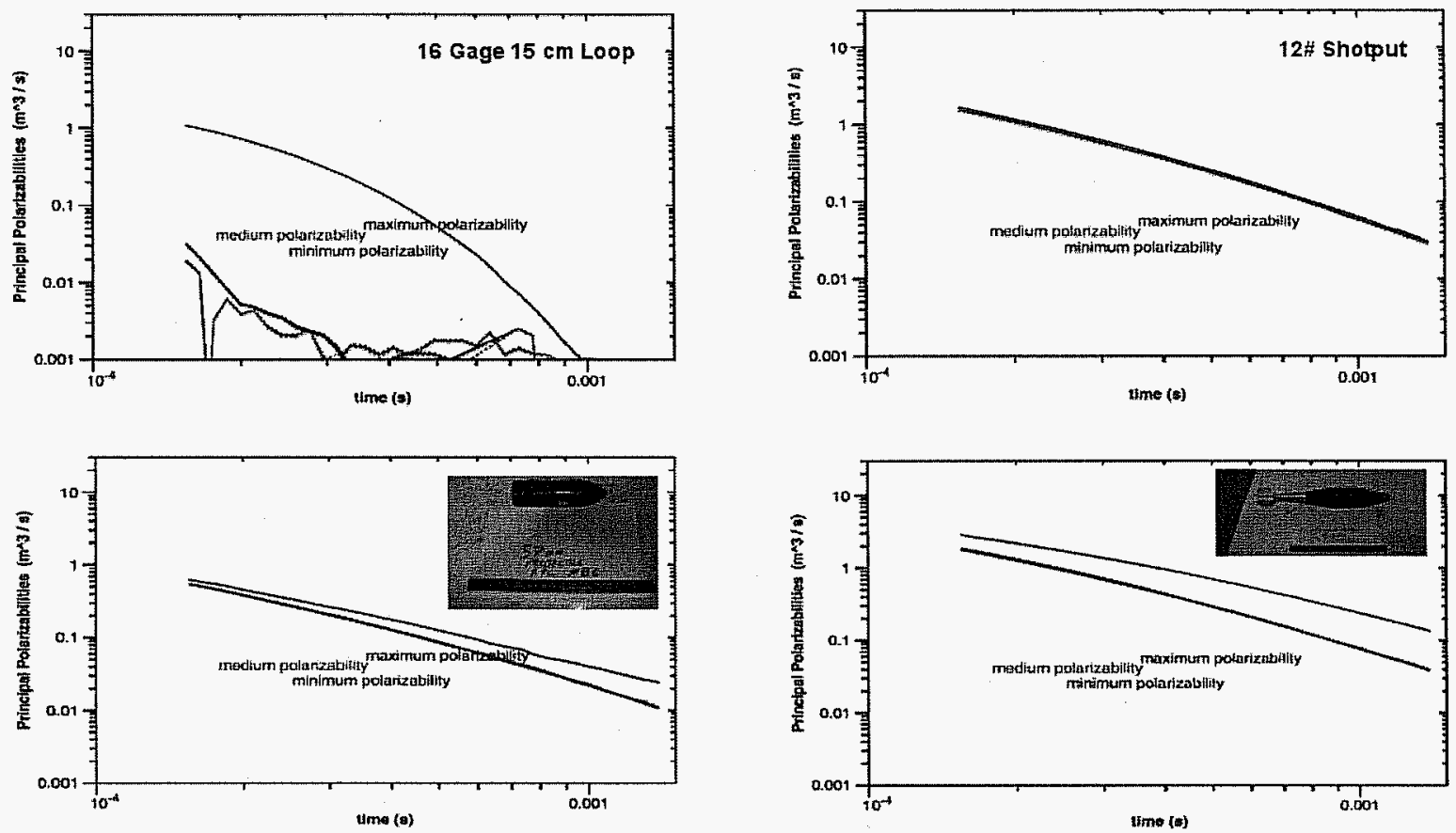

Figure 4: Inversion results for the principal polarizabilities of (a) 16 gage $15 \mathrm{~cm}$ loop, (b) $12-1 \mathrm{~b}$. shot put, (c) $57 \mathrm{~mm}$ projectile, and (d) $81 \mathrm{~mm}$ projectile.

For the data from the Blind Test Grid an object identification program matches measured equivalent dipole polarizabilities to a database of previous measurements of equivalent dipole polarizabilities of known objects and identifies a candidate object as the object(s) corresponding to the closest matching curves from the Calibration Grid and the test pit. Since polarizability curves are prone to greater offsets in overall values than intracurve variations, the distance between measured and database values are measured both in terms of weighted normalized squared offsets between curves and weighted normalized absolute differences between offset curves. Weighting is determined by the variability of these among catalogue responses. The sum of these two measures is used in determining the closest matches.

Figure 5 shows the results from the Blind Test Grid. The logarithm of detection criterion $v$, signal-to-noise weighted index, is plotted in Figure 5a. Large values indicate the clear presence of buried conductive objects. Signal-to-noise levels of 1.58 or higher are significant at a $95 \%$ confidence level, with the background distribution of $v$ modeled as a gamma-distributed random variable with parameters $\alpha=9.38$ and $\lambda=10.25$. The Blind Test Grid detection map is shown in Figure $5 b$. Every cell for which BUD indicates present object is in color, while empty cells are in black. All occupied cells have a time-weighted average signal strength above $9 \times 10^{-12} \mathrm{Vs} / \mathrm{A}$, in response to the $\mathrm{Bz}$ transmitter, or $4.5 \times 10^{-12} \mathrm{Vs} / \mathrm{A}$ in response to either Bx or By transmitters, and the response level is not at a minimum compared to $1 \mathrm{~m}$ forward or backwards along the survey line. The probability of the cell being occupied is based on one minus the probability that the given response level would arise from random fluctuations of the background field in the absence of an object. Out of a total of 400 cells, 230 cells or $57.5 \%$ are occupied. Cells with black plus symbols are empty. $85.1 \%$ of 230 cells have a probability higher than $90 \%$ that they are occupied (red circles), $4.4 \%$ have a probability between $75 \%$ and $90 \%$ (green squares), and $10.5 \%$ have a probability between $30 \%$ and $75 \%$ (blue diamonds). 


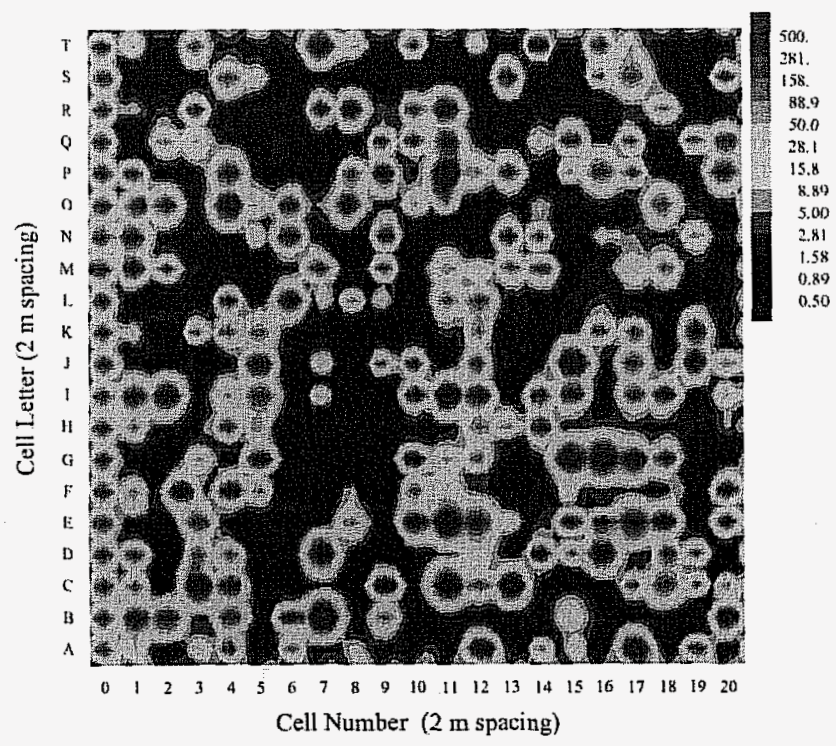

(a)

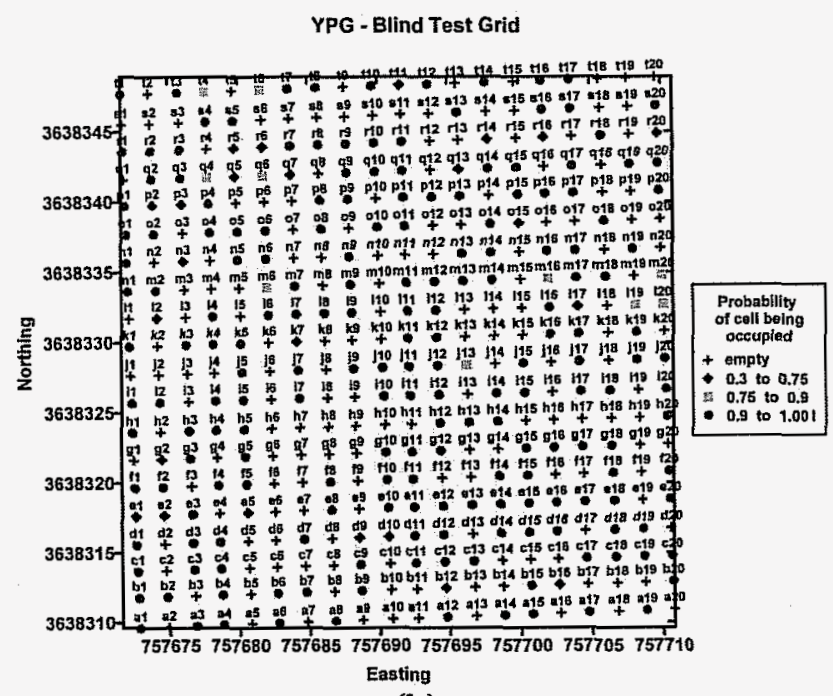

(b)

Figure 5: (a) Logarithm of detection criterion $v$ as a function of cell location in the Blind Test Grid with the response to one row of shot puts delimiting the test area on the left, (b) The Blind Test Grid detection map.

\section{Conclusions}

Our numerical simulations and field data show that complex targets can be accurately detected, located and characterized with a multicomponent transmitter-receiver system. Moreover, there are very clear distinctions between symmetric intact UXO and irregular scrap metal, and BUD can resolve the intrinsic polarizabilities of the target from observations at a single position. The presence or absence of a conductive object in the vicinity of BUD is evaluated using the background signal variation reciprocal weighted average of the absolute signal levels gathered from all eight receivers. Soundings with elevated signal levels are inverted for equivalent dipole polarizability, position, and orientation. The objects are identified by comparison to a catalogue of responses collected over the Calibration Grid and the test pit. In a field survey BUD performed extremely well. It has also proven easy to use and maintain, with transmitter batteries lasting for three hours, and acquisition system batteries lasting for six hours.

\section{Acknowledgments}

This research was funded by the U.S. Department of Defense under SERDP Project \# UX-1225 and ESTCP Project \# UX-0437.

\section{References}

Gasperikova, E., 2003, A new-generation EM system for the detection and classification of buried metallic objects: SEG Expanded Abstracts, pp. 2379-2382. 
Gasperikova E., Smith J.T., Morrison H.F., and Becker A., 2006, UXO Detection and Characterization using new Berkeley UXO Discriminator (BUD), EOS Trans. AGU, 87(36), Jt. Assem. Suppl., Abstract. NS31A-02.

Gasperikova E., Smith J.T., Morrison H.F., and Becker A., 2006, Berkeley UXO Discriminator (BUD) for UXO Detection and Discrimination, Partners in Environmental Technology Technical Symposium \& Workshop, Washington D.C.

Smith, J.T., Allan, W., and Schultz, A., 1994, Inversion of waveform data using an empirical distribution evolutionary algorithm, EOS, 75, p. 457.

Smith, J.T., and Morrison, H.F., 2004, Estimating equivalent dipole polarizabilities for the inductive response of isolated conductive bodies: IEEE Trans. Geosci. Remote Sensing, 42, pp. 12081214.

Smith, J.T., and Morrison, H.F., 2005, Optimizing receiver configurations for resolution of equivalent dipole polarizabilities in situ: IEEE Trans. Geosci. Remote Sensing, 43, pp. 1490-1498.

Smith, J.T., and Morrison, H.F., 2006, Approximating spheroid inductive responses using spheres: Geophysics, 71, pp. G21-G25.

Smith, J.T., Morrison, H.F., and Becker, A., 2004a, Resolution depths for some transmitter receiver configurations: IEEE Trans. Geosci. Remote Sensing, 42, pp. 1215-1221.

Smith, J.T., Morrison, H.F., and Becker, A., 2004b, Parametric forms and inductive response of a permeable conducting sphere: Journ. Environ. Engin. Geosci., 9, pp. 213-216.

Smith, J.T., Morrison, H.F., Doolittle, L.R., and Tseng, H., 2007, Multi-transmitter Multi-receiver Null Coupled Systems for Inductive Detection and Characterization of Metallic Objects, Journal of Applied Geophysics, in press. 


\title{
Print This Notification of Intent to Publish
}

Mail To:

\author{
REPRINTS - Library Group \\ MailStop: 50B-4206 \\ Phone: 510-486-6922 \\ Fax: 510-486-6406 \\ EMAIL: libacq@LBL.GOV
}

Date notification submitted: January 11, 2007

I understand I should notify the Library before sending my paper to the publisher. I am notifying the Library with this form that I expect to incur page charges, illustration/color charges, reprints or any other necessary publication related charges when I submit this paper to the publisher. The paper has not yet been published.

Note: Only the Library is authorized by LBNL to commit LBNL to spend funds on publishing related services from a non-LBNL publisher.

Title: Berkeley UXO Discriminator (BUD)

Author(s): Gasperikova, E.; Smith, J.T.; Morrison, F.; Becker, A.;

Sponsoring LBNL Division: ESD

Contact Section

Employee ID: 016504 Contact Name: Janis E Rasar

MailStop: 90R1116 Address: Bldg. 090 Room 1078E

Phone: 510-486-6696 Fax: 510-486-7714

Email: JRasar@lbl.gov 
rimary Report Contact Erika Gasperikova

econdary Contact (author) Erika Gasperikova

roject ID\# - G5W018

uthors (LBNL authors please initial

$01 / 10 / 2007$

Date Report Completed $\quad 01 / 2007$

\section{itle BERKELEY UXO DISCRIMINATOR (BUD)}

ccess

\Unlimited (open to public viewing)

Limited (contact author for access)

\section{The Proposed Publication is}

Collaboration (published by other institution [not LBNL])

$\square$ Non-technical (doesn't describe any materials, apparatus, or related processes)

$\bigotimes$ Technical (only describes materials, apparatus, or processes already known)

Descriptive (a new material, apparatus, or procedure)
LBNL Topical Report (published by the Berkeley Lab)

Book *Publisher Name

Editor(s)

Book Chapter or Section *

Book Title

Publisher Name

Editor(s)

Conference Publication

\ Abstract*

City, State, Country

Date Needec

\# of Copies Needed

City, State, Country

\# of Copies Needec

Date Needed

Conference Name SAGEEP 2007

Publisher (Conference Sponsor/Host) Environmental and Engineering Geophysical Society

City, State Denver, Colorado

Date (format i.e., May 25-27, 2001) April 2007

Journal *

Journal Name

Journal Publisher

If already accepted: Volume

Thesis for

M.S. $\square$ Ph.D

Issue

Prytation Publication Year

Total \# Pages

Other Reports * (brochures, newsletters, program reports (i.e., DOE Deliverables, AMR, PMR), PUB, LBID)

Type of Report

Demonstration Report

City, State

Pagination

Publisher

Editor(s)

leviewer(s) names

1. Michael Commer

May only need one reviewer)

2.

'rogram/Department Head

unding Source (please check all that apply)

lepartment of Energy

]U.S. DOE, Director, Office of Science (formerly Office of Energy Research)

]U.S. DOE, Assistant Secretary for Energy Efficiency and Renewable Energy

]U.S. DOE, Assistant Secretary for Environmental Management

]U.S. DOE, Assistant Secretary for Environment, Safety and Health

]U.S. DOE, Assistant Secretary for Fossil Energy

JU.S. DOE, Office of Civilian Radioactive Waste Management

]U.S. DOE, LDRD

rogram

INuclear Waste

JEnergy Resources

Environmental Remediation

Fundamental and Exploratory Research

JClimate Change and Carbon Management

Ext. 4930

Ext. 4930 